\title{
GLOBAL STRUCTURE OF THE FALICOV-KIMBALL MODEL GROUND STATE PHASE DIAGRAM
}

\author{
J. LACII, R. LYŻWA \\ Institute of Low Temperature and Structure Research, Polish Academy of Sciences \\ P.O. Box 937, 50-950 Wrocław, Poland \\ AND J. JĘDRZEJEWSKI \\ Institute of Theoretical Physics, University of Wroclaw \\ Pl. Maxa Borna 9, 50-204 Wrocław, Poland \\ (Received February 23, 1993; in final form May 31, 1993)
}

The two-state spinless Falicov-Kimball model on a one-dimensional lattice is studied by means of well-controlled numerical procedures. Restricted phase diagrams in the grand-canonical ensemble and at zero temperature are constructed. The evolution of these phase diagrams, as the interaction parameter $U$ is varied, including the band structures corresponding to configurations of localized particles (ions) and densities of mobile particles (electrons), is monitored. The changes observed enable us to draw conclusions for the global structure of the pliase diagram and to make a qualitative plot of such a diagram. Particular attention is paid to the possibility of conductor-insulator transitions. The results obtained suggest that the system is an insulator for all $U$ only if the electron and ion densities $\rho_{\mathrm{e}}, \rho_{\mathrm{i}}$ satisfy the conditions: $\rho_{\mathrm{e}}+\rho_{\mathrm{i}}=1,\left|\rho_{\mathrm{i}}-\frac{1}{2}\right| \leq \delta<\frac{1}{2}$. For all other pairs $\left(\rho_{\mathrm{e}}, \rho_{\mathrm{i}}\right)$ there is a critical $U$ at which the system undergoes a conductor-insulator transition.

PACS numbers: 75.30.Mb, 71.30.th

\section{Introduction}

The work reported in this paper has been inspired by the recent results due to Freericks and Falicov [1], Gruber [2], Lemberger [3] and Gruber et al. [4]. It is also a continuation of our earlier work $[5,6]$. All the papers mentioned are concerned with various properties of the phase diagram of the two-state spinless Falicov-Kimball model. Here we make an attempt to obtain a global qualitative image of this diagram, paying special attention to the possibility of conductor-insulator transitions. 
In fact, the model considered is a result of the attempt to describe such transitions in intermediate-valence compounds of rare earths [7].

Generally speaking, we are interested in correlation effects in fermion lattice gases. This is, of course, a huge subject with numerous approaches and models (see for instance review papers by Khomskii [8] and Czycholl [9]). To present the system we are going to discuss, without going into details, we can say that this is an interacting system that can be thought of as a simplest step beyond the band theory. The interaction between the electrons is introduced by comparing the ground state energies of band systems in different external potentials. If our electrons can only move on a lattice, do not carry the spin and the external potential assumes only two values, we arrive at the most popular version of the system introduced above, the spinless Falicov-Kimball model. The two values of the external potential at a site $x$ of a lattice $\Lambda$ can be interpreted as presence and absence of an ion at $x$ and different potentials as different configurations of localized particles called ions. This interpretation provides us with a point of view that is complementary to the above one: by comparing band systems in different external potentials we introduce the effective interaction between the ions. It is this interaction, inherently many-body and long-range one [10], which is the subject of our interest.

The Falicov-Kimball model can be related to the celebrated Ilubbard model $[10,12]$. It has however many interpretations of its own $[1,8,10]$ interesting enough to motivate intensive investigations of this model. In one of the interpretations proposed by Khomskii [8], Kennedy and Lieb [10] and adopted here it is a model of crystalline formation. As proved by Kennedy and Lieb [10] and Brandt and Schmidt [11] the effective interaction between the ions, induced by the direct interaction with mobile electrons, leads to the formation of periodic structures of the ions (superlattices). Another interpretation that is sound from the point of view of solid state physics, is in terms of mobile $s$-electrons and localized $f$-electrons in rare-earth compounds. This makes the model relevant for understanding mixed-valence phenomena $[7,8]$.

In this paper we study general properties of the ground-state phase diagram of the spinless Falicov-Kimball model on the one-dimensional lattice. In distinction to the papers quoted above we consider the system in the grand canonical ensemble. The reasons for this choice are given in the next section. The phase diagram of this system is of great importance for the theory of strongly correlated fermion systems. It contains the complete information about the equilibrium properties of this system which can eventually be used to study more specific questions. We leave the examination of the implications for particular phenomena such as transitions to mixed-valence states to a forthcoming paper. We think that it is worth to mention here that a deep understanding of the one-dimensional case might be very helpful for understanding of the much more complicated 2- and 3-dimensional cases. Some interesting results in this direction were obtained recently by Freericks [19].

The peculiar structure of the Falicov-Kimball model considered here makes it amenable to rigorous studies $[2-4,10,11,13]$. We shall comment on these results in due course, in the sequel.

The paper is organized as follows. We set notations, define the IIamiltonian 
and auxiliary notions in Sec. 2. Then in Sec. 3 we consider a coarse structure of the restricted ground state phase diagrams. $\mathrm{A}$ fine structure is considered in the following two sections, the case of large values of the coupling $U$ in Sec. 4 while the evolution with decreasing $U$ and the case of small $U$ in Sec. 5. The hypotheses for the complete phase diagram, which are put forward in Secs. 4 and 5, are summarized in Sec. 6. In this section we consider also the consequences of these hypotheses with respect to conductor-insulator transitions.

\section{The Hamiltonian and auxiliary notions}

We assume from now on that the underlying lattice $A$ is one-dimensional, $\Lambda \subset \mathbb{Z}$ (ZZZ being integers), and the electrons can hope from a site to its nearest neighbors (this implies an extra symmetry of the model [10]).

The grand-canonical IIamiltonian has the form

$$
H(S)=\sum_{x, y \in \Lambda} h_{x y}(S) a_{x}^{*} a_{y}-\mu_{\mathrm{e}} N_{\mathrm{e}}-\left(\mu_{\mathrm{i}}+U\right) N_{\mathrm{i}}(S) .
$$

In Eq. (1) $a_{x}^{*}, a_{x}$ stand for the creation and annihilation operators respectively of a spinless electron at the site $x \in \Lambda$ and

$$
h_{x y}(S)=t_{x y}+U s_{x} \delta_{x y},
$$

where

$$
\begin{aligned}
& t_{x y}= \begin{cases}1 & \text { if } y=x \pm 1 \text { and } \\
0 & \text { otherwise, }\end{cases} \\
& s_{x}= \begin{cases}1 & \text { if } x \text { is occupied by an ion, } \\
-1 & \text { otherwise, }\end{cases}
\end{aligned}
$$

are the matrix elements of the single-particle operator

$$
h(S)=T+U S
$$

acting in the $|\Lambda|$-dimensional IIilbert space $(|\Lambda|$ being the number of sites in $\Lambda$ ). The operators of the total electron and ion numbers read

$$
N_{\mathrm{e}}=\sum_{x \in \Lambda} a_{x}^{*} a_{x}, \quad N_{\mathrm{i}}(S)=\sum_{x \in \Lambda}\left(1+s_{x}\right) / 2
$$

and the corresponding chemical potentials are denoted by $\mu_{e}, \mu_{i}$, respectively. We have chosen the scale of the chemical potentials in such a way that (1) is hole-particle invariant at $\mu_{\mathrm{e}}=\mu_{\mathrm{i}}=0$. It turns out that the sign of the coupling $U$ between the ions and electrons is immaterial, since both cases (repulsive and attractive) are related by a unitary transformation [4]. We choose $U>0$.

The effective interaction between the ions can easily be expressed in terms of $h(S)[10,11]$. In the limit of zero temperature and infinite volume, $\Lambda \nearrow \mathbb{Z}$, the energy density corresponding to this interaction assumes the form

$$
e\left(\mu_{\mathrm{e}}, \mu_{\mathrm{i}} ; S\right)=-\int_{-\infty}^{\mu_{\mathrm{e}}} \mathrm{d} \mu \rho_{\mathrm{e}}(\mu ; S)-\left(\mu_{\mathrm{i}}+U\right) \rho_{\mathrm{i}}(S),
$$


where $\rho_{\mathrm{e}}\left(\mu_{\mathrm{e}} ; S\right)$ is the electron density at the Fermi level $\mu_{\mathrm{e}}$ when the ion configuration is $S$ and $\rho_{\mathrm{i}}(S)$ is the ion density. Apparently the effective interaction between the ions is many-body and long-range one.

In this paper we study properties of the Falicov-Kimball effective interaction by means of restricted phase diagrams (r.p.d.'s) at zero temperature. This sort of approach has already been used by ourselves $[5,6]$ and by Freericks and Falicov [1]. In our earlier work $[5,6]$ a very specific set of periodic two-dimensional configurations with $\rho_{\mathrm{i}}=\frac{1}{2}$ and a sequence of $\rho_{\mathrm{e}}\left(\rho_{\mathrm{e}}=1 / 2^{n}, n=1,2, \ldots\right)$ was considered. Later on, a more systematic study, in the canonical ensemble with $\rho_{\mathrm{i}}=\frac{1}{2}, \frac{2}{3}$ and $\rho_{\mathrm{e}}$-arbitrary, for the set of all one-dimensional periodic configurations whose period did not exceed 8 (10 configurations), was performed [1]. Contrary to the earlier papers, here we consider the system in the grand-canonical ensemble. This enables us to consider simultaneously a variety of particle densities $\rho_{\mathrm{e}}, \rho_{\mathrm{i}}$. Due to the concavity of the energy density (5) in $\left(\mu_{\mathrm{e}}, \mu_{\mathrm{i}}\right)$, unlike in the canonical ensemble [1], we do not have to take into account mixed phases. Moreover, we control the band structures of the ion configurations, determining whether they are conductors or insulators for a given Fermi level or electron density. The latter notions are understood in the sense of the standard band theory.

To this end we chose the set $\mathcal{S}_{6}$ of all one-dimensional periodic configurations on $\mathbb{Z}$ whose period is not greater than 6 . The set $\mathcal{S}_{6}$ consists of 22 different, that is, not related by lattice translations, classes of configurations, see Fig. 1a (in the sequel we shall use the terms class and configuration as synonyms). Then the functions $\mu \rightarrow \rho_{\mathrm{e}}(\mu ; S), S \in \mathcal{S}_{6}$, were determined. At zero temperature and infinite volume these functions are piecewise differentiable with a finite number of points without a tangent. The points of non-differentiability mark the band edges. Between the band edges, in the bands, the functions $\mu \rightarrow \rho_{\mathrm{e}}(\mu ; S)$ were determined exactly in terms of elementary functions [14]. Moreover, the polynomial equations (of the orders of the periods of the configurations), whose roots coincide with the band edges, were derived. Ilaving determined numerically the band edges, we calculated, by numerical integration, the functions (5). We note that the numerical tasks which were performed are good numerical problems in the sense that a high accuracy in the final results can be achieved. The limitation of the periods of the configurations considered to 6 enabled us to use factorization properties of the polynomials mentioned above. Finally, at each point $\left(\mu_{\mathrm{e}}, \mu_{\mathrm{i}}\right)$ of the plane of the chemical potentials we found the set $G\left(\mu_{\mathrm{e}}, \mu_{\mathrm{i}}\right)$ of those ion configurations from $\mathcal{S}_{6}$ that minimize the function $S \rightarrow e\left(\mu_{\mathrm{e}}, \mu_{\mathrm{i}} ; S\right)$, i.e. the set of the $\mathcal{S}_{6}$-restricted ground state configurations ( $\mathcal{S}_{6}$-r.g.s.c.'s).

As a result, the whole plane of the chemical potentials was partitioned into connected sets with all points $\left(\mu_{\mathrm{e}}, \mu_{\mathrm{i}}\right)$ in a set having the same set $G$. This task was facilitated by the inversion symmetry of the phase diagram [4]: if $S \in G\left(\mu_{\mathrm{e}}, \mu_{\mathrm{i}}\right)$ with densities $\rho_{\mathrm{e}}\left(\mu_{\mathrm{e}} ; S\right)$ and $\rho_{\mathrm{i}}(S)$, then $S \in G\left(-\mu_{\mathrm{e}},-\mu_{\mathrm{i}}\right)$ with densities $1-\rho_{\mathrm{e}}\left(\mu_{\mathrm{e}} ; S\right)$ and $1-\rho_{\mathrm{i}}(S)$, respectively.

The procedure described above was repeated for a sequence of values of the interaction parameter $U$. There is a characteristic value $U_{\mathrm{sl}}$ of $U$ that fixes the scale for $U$. In our case $U_{\mathrm{sl}}=2$. If $A$ is the $\nu$-dimensional hypercubic lattice, then $U_{\mathrm{sl}}=2 \nu$. Note however that $U_{\mathrm{sl}}$ depends on the parametrization of $H(S)$. For 

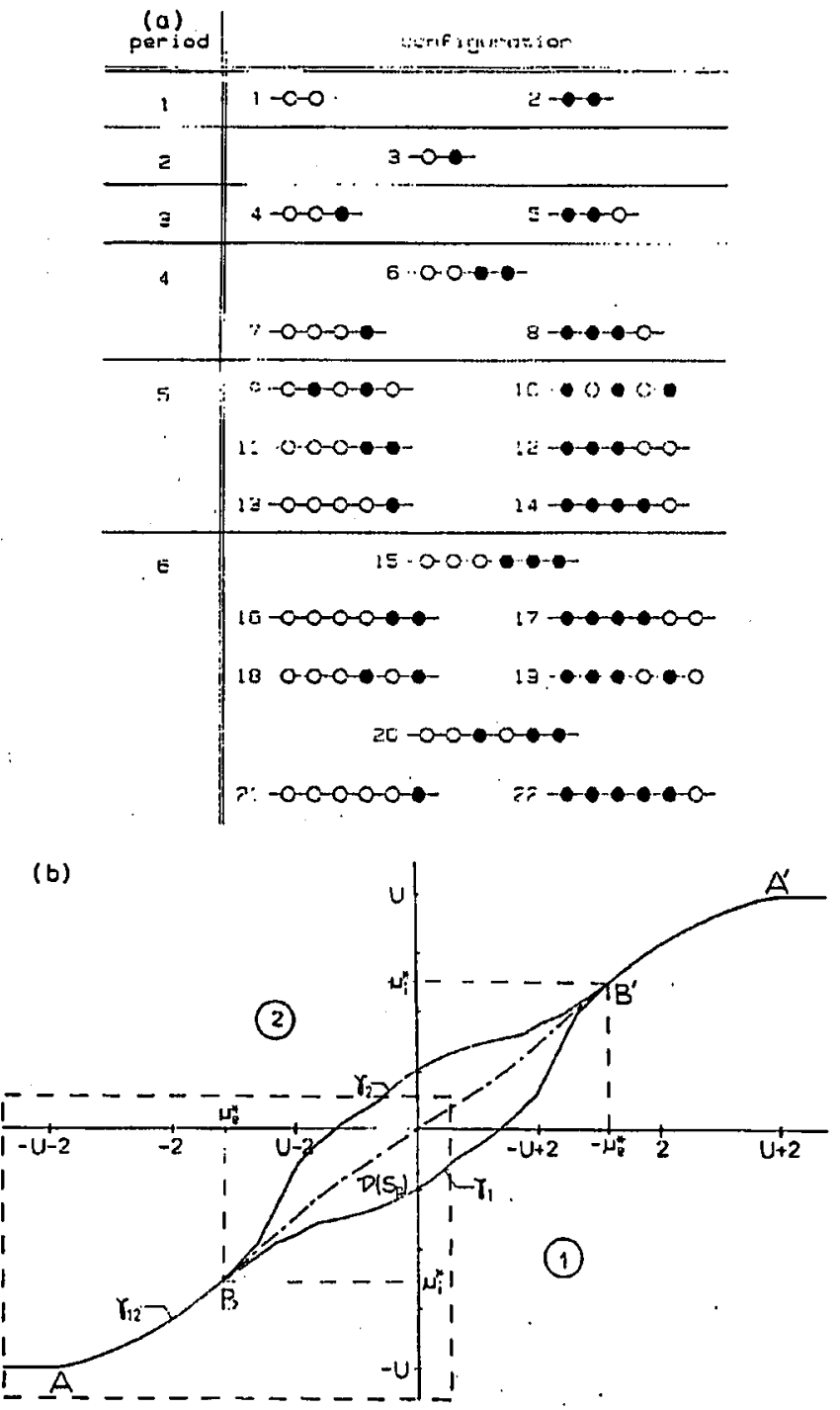

Fig. 1. Guide to the phase diagrams shown in Figs. 2-6. (a) The distribution of the ions, marked by the black circles, in the elementary cells of the periodic configurations whose period is not greater than 6 . (b) General structure of the $\mathcal{S}_{6}$-restricted phase diagrams. The connected region $\mathcal{D}\left(\mathcal{S}_{6}\right)$, bounded by the curves $\gamma_{1}$ and $\gamma_{2}$, is the union of the domains of translationally noninvariant configurations. The curve $\gamma_{1,2}$, where the energies of $c .1$ and c.2 are equal, consists of three segments: $A B, B B^{\prime}$ (the dash-dotted

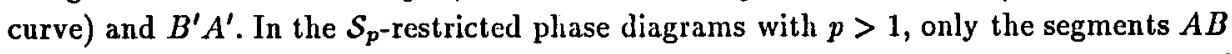
and $B^{\prime} A^{\prime}$ represent domains of the phase diagram: at these segments the coexistence of c. 1 and c. 2 takes place. In Figs. 2-6 we display only the part of the $\mathcal{S}_{6}$-restricted phase diagram which is contained in the rectangle marked by the dashed lines. The remaining part can be reconstructed by the inversion about the origin. 
$U>U_{\text {sl }}$ (we say $U$ large), the bands of any configuration $S$ are split into a lower group and an upper group by the gap $\left[-U+U_{\mathrm{sl}}, U-U_{\mathrm{sl}}\right]$. The lower groúp is contained in the unique band of configuration 1 (c.1) with no ions (see Fig. 1a), i.e. in $\left[-U-U_{\mathrm{sl}},-U+U_{\mathrm{sl}}\right]$, while the upper group is in the unique band of c.2 with all sites occupied, i.e. in $\left[U-U_{\mathrm{sl}}, U+U_{\mathrm{sl}}\right]$. Moreover, the lower group can accommodate exactly $1-\rho_{\mathrm{i}}(S)$ electrons [4].

In Figs. 2-6 we display the r.p.d.'s for the values of $U: 0.1,0.5,1.5,2.5,4$, ranging from $0.05 U_{\mathrm{sl}}$ to $2 U_{\mathrm{sl}}$ (a larger set of diagrams can be found in our preprint
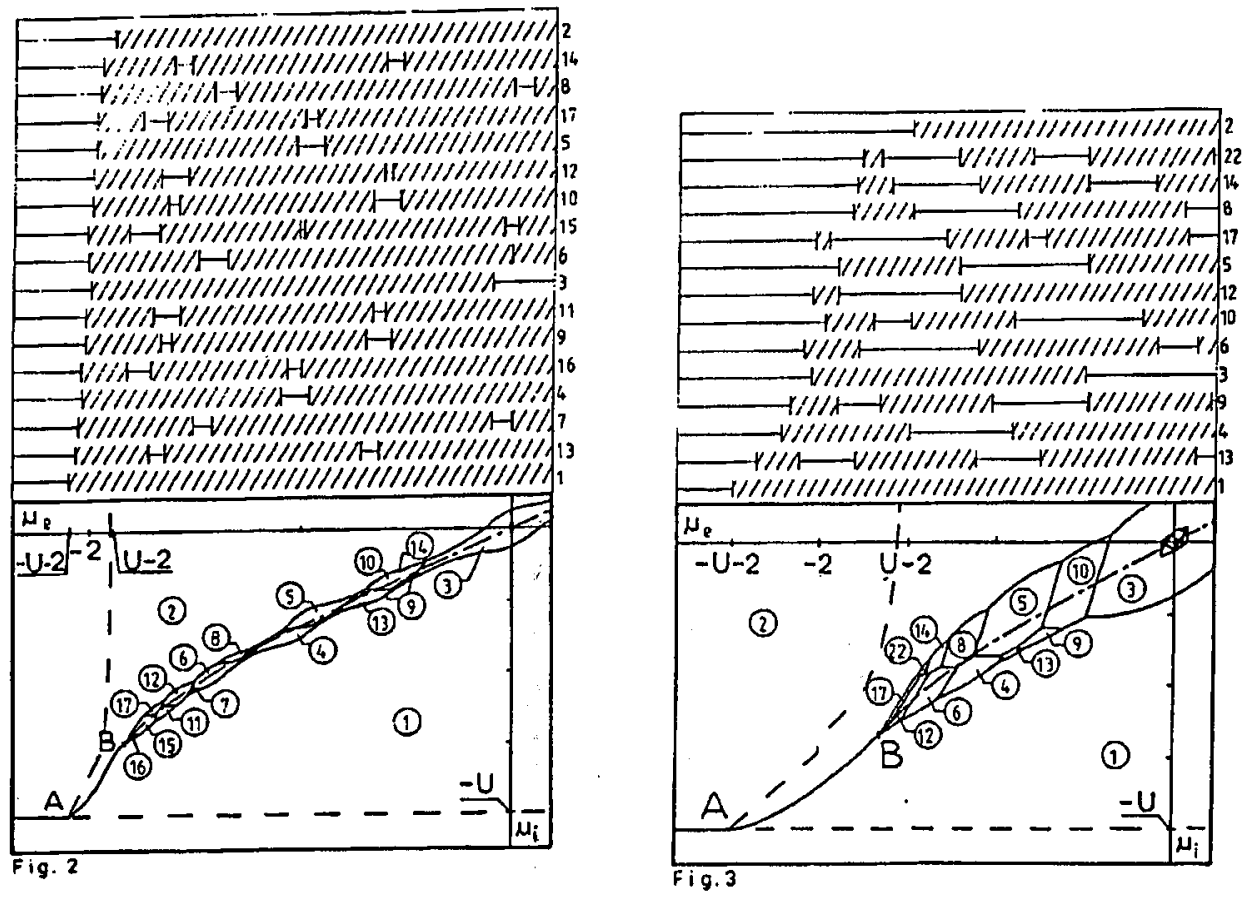

Fig. 2. $\mathcal{S}_{6}$-restricted phase diagram for $U=0.1$. The dashed lines represent the boundary of the region that certainly contains $\mathcal{D}\left(\mathcal{S}_{p}\right)$. For this $U$ and on the scale assumed the lower bound for the domain $d_{3}\left(\mathcal{S}_{\infty}\right)$ is the spot at the origin. For larger $U$ this bound is displayed as a cross-latched polygon. But for $U=6$, on the scale assumed, this polygon degenerates into a thick line segment in the center of the diagram. The continuous lines represent the boundaries of the domains where particular classes of configurations, shown in the attached circles, are the ground state configurations. The dash-dotted curve is the continuation of the lines of the coexistence of c.1 and c.2. In the upper part of the figure we display the positions of the bands in the spectrum of those configurations that appear in the phase diagram. The bands are represented by the hatched intervals. In the q-th gap, counting from the bottom of the spectrum of a periodic configuration with the period $p$, the electron density $\rho_{\mathrm{e}}=q / p$.

Fig. 3. $\mathcal{S}_{6}$-restricted phase diagram for $U=0.5$. See Figs. 1 and 2 for the key to the legend. 
[20]). In these figures we show also the positions of the bands in the spectrum of the r.g.s.c.'s. The electron density in the gaps can easily be fixed [14] (see the caption of Fig. 2). Actually we show only a part of these diagrams (cf. Fig. 1b). The other part can be obtained by the symmetry.

To facilitate the discussion of r.p.d.'s (Figs. 2-6) in the sequel, let us introduce some notation and terminology (that, to some extent, is a generalization of the notation and terminology introduced above). The r.p.d. with the set $\mathcal{S}$ of the ion configurations we call the $\mathcal{S}$-r.p.d. The set $\mathcal{S}$ which consists of all the configurations on $\mathbb{Z}$ whose period is not greater than $p$ is denoted by $\mathcal{S}_{p}$ (thus $\mathcal{S}_{p+1} \supset \mathcal{S}_{p}$ ). By $\mathcal{S}_{\infty}$ we denote the set of all the ion configurations on $\mathscr{Z}$ (hence the complete phase diagram coincides with the $\mathcal{S}_{\infty}$-r.p.d.). The union of all points $\left(\mu_{\mathrm{e}}, \mu_{\mathrm{i}}\right)$ where the class $x$ is the only $\mathcal{S}$-r.g.s.c., i.e. $G=\{x\}$, is called the class $x$ (c. $x$ )-domain and is denoted by $d_{x}(\mathcal{S})$. The c.x-domain may not be connected. If $\mathcal{S}^{\prime} \supset \mathcal{S}$, then $d_{x}(\mathcal{S}) \supset d_{x}\left(\mathcal{S}^{\prime}\right)$, i.e. $d_{x}(\mathcal{S})$ is an upper bound (in the sense of set inclusion) for $d_{x}\left(\mathcal{S}_{\infty}\right)$. Any $\mathcal{S}_{p}$-r.p.d. has to satisfy the thermodynamic stability conditions which reflect the concavity of the grand-canonical potential with respect to the pair of the chemical potentials $\left(\mu_{\mathrm{e}}, \mu_{\mathrm{i}}\right)$. In particular, if $\rho_{\mathrm{e}}\left(\mu_{\mathrm{e}}, \mu_{\mathrm{i}}\right)$ and $\rho_{\mathrm{i}}\left(\mu_{\mathrm{e}}, \mu_{\mathrm{i}}\right)$ stand for the electron and ion densities at the point $\left(\mu_{\mathrm{e}}, \mu_{\mathrm{i}}\right)$ of an $\mathcal{S}_{p}$-r.p.d., then the
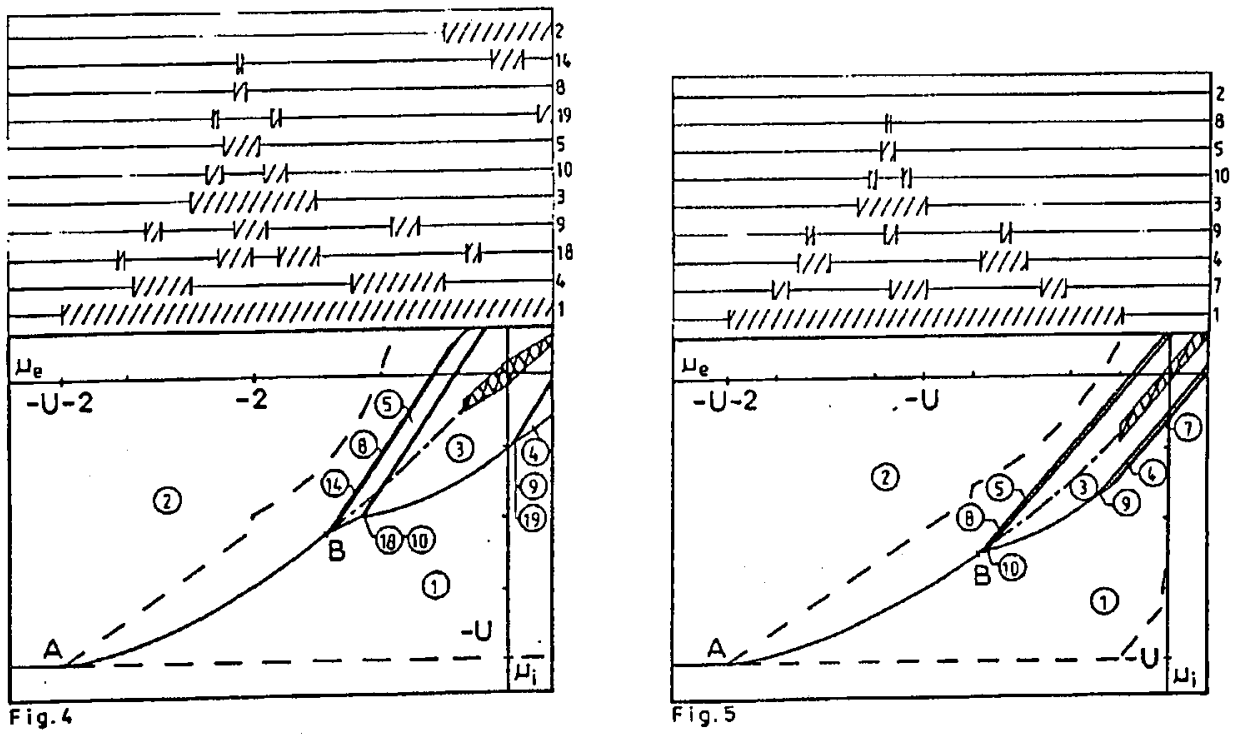

Fig. 4. $S_{6}$-restricted phase diagram for $U=1.5$. See Figs. 1 and 2 for the key to the legend. The two thick lines represent collections of narrow domains which extend along these lines. The classes of configurations which are the ground state configurations in these domains are shown in the attached clusters of circles. The order of the labels from right to left (in the horizontal cluster) or upwards (in the vertical cluster) corresponds to the order in which a vertical line intersects these domains when we move upwards. Fig. 5. $\mathcal{S}_{6}$-restricted phase diagram for $U=2.5$. Sec Figs. 1 and 2 for the key to the legend. 

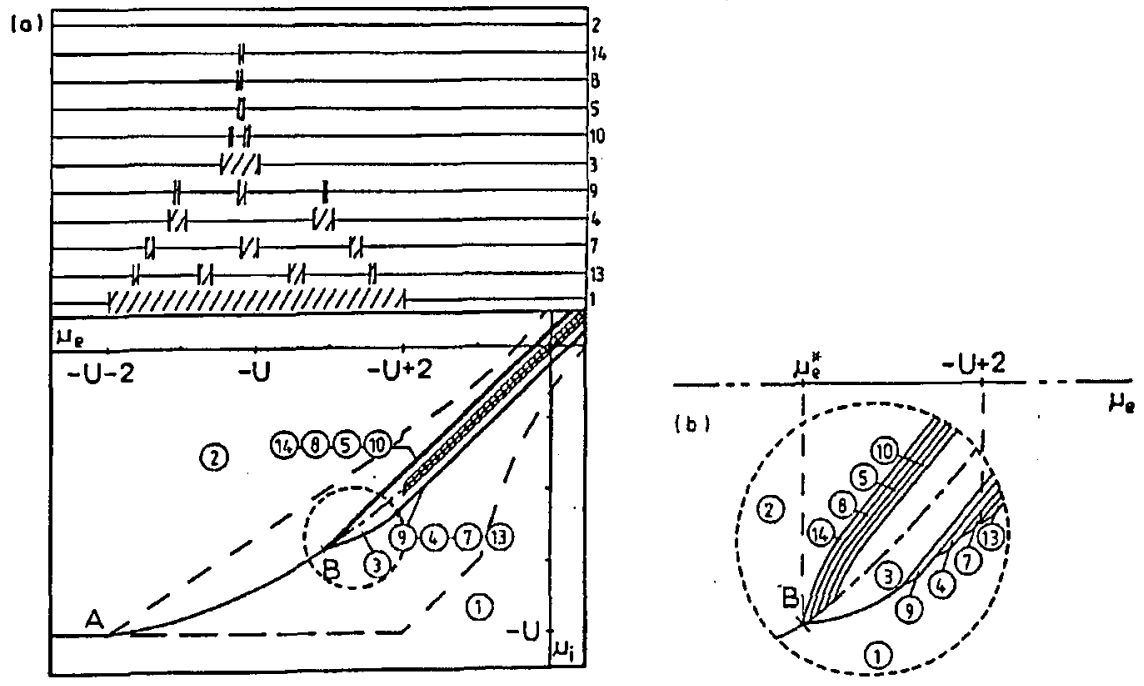

Fig. 6. (a) $\mathcal{S}_{6}$-restricted phase diagram for $U=4$. See Figs. 1,2 , and 4 for the key to the legend. (b) The magnified view of the area in Fig. 6b shown in the dashed circle; the qualitative picture.

functions $\mu_{\mathrm{e}} \rightarrow \rho_{\mathrm{e}}\left(\mu_{\mathrm{e}}, \mu_{\mathrm{i}}\right)$ and $\mu_{\mathrm{i}} \rightarrow \rho_{\mathrm{i}}\left(\mu_{\mathrm{e}}, \mu_{\mathrm{i}}\right)$ have to be non-decreasing on their domains [4].

\section{The coarse structure}

The general shape of the complete phase diagram and any $\mathcal{S}$-r.p.d. is determined by the set $\gamma_{1,2}$ on which the energies of the only two translationally invariant configurations (c.1 and c.2) are equal. It turns out [4] that this set is a curve with the parametric representation $\left(\mu_{\mathrm{e}}, f\left(\mu_{\mathrm{e}}\right)\right)$, where $f$ is the function equal to $-U$ below the band of c. 1 , strictly increasing between the lower edge of the band of $c .1$ and the upper edge of the band of c.2, and equal to $U$ above the band of c.2. In one dimension this function can easily be expressed in terms of elementary functions. The nontrivial part of this function, i.e. in the interval $[-U-2, U+2]$ reads

$$
\frac{\mu_{\mathrm{e}}^{-}}{2}+\frac{1}{\pi}\left\{\mu_{\mathrm{e}}^{+} \arcsin \frac{\mu_{\mathrm{e}}^{+}}{2}+\left[4-\left(\mu_{\mathrm{e}}^{+}\right)^{2}\right]^{1 / 2}\right\}
$$

if $-U-2<\mu_{\mathrm{e}} \leq U-2$ and $U<2$ or if $-U-2<\mu_{\mathrm{e}} \leq-U+2$ and $U \geq 2$,

$$
\frac{1}{\pi}\left\{\mu_{\mathrm{e}}^{+} \arcsin \frac{\mu_{\mathrm{e}}^{+}}{2}-\mu_{\mathrm{e}}^{-} \arcsin \frac{\mu_{\mathrm{e}}^{-}}{2}+\left[4-\left(\mu_{\mathrm{e}}^{+}\right)^{2}\right]^{1 / 2}-\left[4-\left(\mu_{\mathrm{e}}^{-}\right)^{2}\right]^{1 / 2}\right\}
$$

if $U-2<\mu_{\mathrm{e}} \leq-U+2$ and $U<2$,

$$
\begin{gathered}
\mu_{\mathrm{e}} \\
\text { if }-U+2<\mu_{\mathrm{e}} \leq U-2 \text { and } U \geq 2, \\
\frac{\mu_{\mathrm{e}}^{+}}{2}-\frac{1}{\pi}\left\{\mu_{\mathrm{e}}^{-} \arcsin \frac{\mu_{\mathrm{e}}^{-}}{2}+\left[4-\left(\mu_{\mathrm{e}}^{-}\right)^{2}\right]^{1 / 2}\right\}
\end{gathered}
$$


if $-U+2<\mu_{\mathrm{e}} \leq U+2$ and $U<2$ or if $U-2<\mu_{\mathrm{e}} \leq U+2$ and $U \geq 2$, where $\mu_{\mathrm{e}}^{ \pm}:=\mu_{\mathrm{e}} \pm U$.

The general structure of the $\mathcal{S}_{p}$-r.p.d. is shown in Fig. 1b. On the $\mathcal{S}_{1}$-r.p.d. the curve $\gamma_{1,2}$ is the place where the coexistence of c.1 and c.2 occurs. The region above $\gamma_{1,2}$ is the c.2-domain while the one below $\gamma_{1,2}$ is the c.1-domain. When $\mathcal{S}_{1}$ is replaced by $\mathcal{S}_{p}, p \geq 2$, the curve $\gamma_{1,2}$ splits into two branches at the point $\left(\mu_{\mathrm{e}}^{*}\left(\mathcal{S}_{p} ; U\right), \mu_{\mathrm{i}}^{*}\left(\mathcal{S}_{p} ; U\right)\right)$, where $-U-2<\mu_{\mathrm{e}}^{*}\left(\mathcal{S}_{p} ; U\right)<\min (0,-U+2)$, $-U<\mu_{\mathrm{i}}^{*}=f\left(\mu_{\mathrm{e}}^{*}\right)<0$. These branches merge into $\gamma_{1,2}$ at the inversion symmetric point $\left(-\mu_{\mathrm{e}}^{*},-\mu_{\mathrm{i}}^{*}\right)$. For $\mu_{\mathrm{e}} \in\left[\mu_{\mathrm{e}}^{*},-\mu_{\mathrm{e}}^{*}\right]$ the upper branch $\gamma_{2}$ stays above $\gamma_{1,2}$ while the lower branch $\gamma_{1}$ stays below $\gamma_{1,2}$. The branches $\gamma_{1}$ and $\gamma_{2}$ constitute the new boundaries of the c.1-domain and c.2-domain, respectively. The connected region $\mathcal{D}\left(\mathcal{S}_{p}\right)$ surrounded by $\gamma_{1}$ and $\gamma_{2}$ is the union of the domains whose sets $G$ do not contain the translationally invariant configurations. On the horizontal parts of $\gamma_{1,2}, G=\mathcal{S}_{p}$. The line of the coexistence of c.1 and c.2 is thus reduced to the two non-horizontal segments of $\gamma_{1,2}$ which are not covered by $\mathcal{D}\left(\mathcal{S}_{p}\right)$ (that is $-U-2 \leq \mu_{\mathrm{e}} \leq \mu_{\mathrm{e}}^{*}\left(\mathcal{S}_{p} ; U\right)$ and $\left.-\mu_{\mathrm{e}}^{*}\left(\mathcal{S}_{p} ; U\right) \leq \mu_{\mathrm{e}} \leq U+2\right)$.

Looking at the $\mathcal{S}_{p}$-r.p.d. and how they change when $\mathcal{S}_{p}$ and (or) $U$ are varied, we see that they possess two types of structures: a coarse structure and a fine one. The coarse structure, exhibited by the extension of $\mathcal{D}\left(\mathcal{S}_{p}\right)$ and the size of the two segments of $\gamma_{1,2}$ where c. 1 and c.2 coexist, is the result of the competition between the translationally invariant configurations and those that are not translationally invariant. The fine structure, that is, the partition of $\mathcal{D}\left(\mathcal{S}_{p}\right)$ into domains of various classes of configurations, is due to the competition of the translationally noninvariant configurations among themselves.

First, we concentrate on the coarse structure. The $\mathcal{S}_{p}$-r.p.d.'s provide us with an increasing sequence of lower bounds for $\mathcal{D}\left(\mathcal{S}_{\infty}\right): \mathcal{D}\left(\mathcal{S}_{p}\right) \subset \mathcal{D}\left(\mathcal{S}_{p+1}\right) \subset \mathcal{D}\left(\mathcal{S}_{\infty}\right)$. Our data suggest that for $U>U_{\text {sl }}$, already $d_{3}\left(\mathcal{S}_{2}\right)$ is a fairly good approximation to $\mathcal{D}\left(\mathcal{S}_{\infty}\right)$. The domains $\mathcal{D}\left(\mathcal{S}_{p}\right), p \geq 2$, follow closely the curve $\gamma_{1,2}$ in the sense that the ratio of the maximal width of $\mathcal{D}\left(\mathcal{S}_{p}\right)$, measured along the directions perpendicular to $\gamma_{1,2}$, to the length of $\gamma_{1,2} \cap \mathcal{D}\left(\mathcal{S}_{p}\right)$ is much smaller than 1 . When $U \rightarrow 0$ or $U \rightarrow \infty$, this ratio tends to zero, since the width of $\mathcal{D}\left(\mathcal{S}_{p}\right)$ tends to zero while the length along $\gamma_{1,2}$ remains bounded from below by a non-zero constant. The minimal rectangle $R$ that contains the non-horizontal segment of $\gamma_{1,2}$, i.e., $-U-2<\mu_{\mathrm{e}}<U+2$ and $-U<\mu_{\mathrm{i}}<U$, is an upper bound for $\mathcal{D}\left(\mathcal{S}_{\infty}\right)$, and hence for any $\mathcal{D}\left(\mathcal{S}_{p}\right)$. In the complement of this rectangle the r.p.d.'s are known for any $\mathcal{S}$ : above $\gamma_{1,2}, G$ consists of c.2, below $\gamma_{1,2}, G$ consists of c.1 while on the horizontal segments (where $f$ is constant) $G=\mathcal{S}$ [1]. Some better than $R$ bounds for $\mathcal{D}\left(\mathcal{S}_{\infty}\right)$ were also constructed $[4,13]$ (in Figs. 2-10 these bounds are shown by dashed lines). The $\mathcal{S}_{p}$-r.p.d.'s suggest a still better upper bound for $\mathcal{D}\left(\mathcal{S}_{p}\right)$ : clearly the best rectangular upper bound for $\mathcal{D}\left(\mathcal{S}_{p}\right)$ is the rectangle built on the two points where the coexistence lines of c.1 and c.2 touch $\mathcal{D}\left(\mathcal{S}_{p}\right)$. Since $\mu_{\mathrm{e}} \cdot\left(\mathcal{S}_{p} ; U\right)$ depends strongly on $\mathcal{S}_{p}$, it is difficult to predict the location of this bound in the $\mathcal{S}_{\infty}$-case, for a given $\hat{U}$. However, when $U$ is varied, some trends, independent of $p(p \leq 6)$, are clearly seen. Therefore, it is likely that these features hold in the $\mathcal{S}_{\infty}$-case. In particular, it seems that $\mu_{\mathrm{e}}^{*}\left(\mathcal{S}_{p} ; U\right)$ tends to the upper edge of the band of c.1 as $U$ grows to infinity and $\mu_{\mathrm{e}}^{*}\left(\mathcal{S}_{p} ; U\right)$ tends to the lower edge of 
this band as $U$ decreases towards zero. lior $U>0, \mu_{\mathrm{e}}^{*}\left(\mathcal{S}_{p} ; U\right)$ is between (does not coincide with) the band edges of c.1. Moreover, for sufficiently small $U$ it is conceivable that $\mu_{\mathrm{e}}^{*}\left(\mathcal{S}_{\infty} ; U\right)$ enters the band of c.2, i.e. $\mu_{\mathrm{e}}^{*}\left(\mathcal{S}_{\infty} ; U\right)>U-2$ (cf. Fig. 2); correspondingly $-\mu_{\mathrm{e}}^{*}\left(\mathcal{S}_{\infty} ; U\right)<-U+2$.

The physical meaning of the above observations is, perhaps, better revealed if they are translated to the canonical ensemble, where the state of the system is specified by a point $\left(\rho_{\mathrm{e}}, \rho_{\mathrm{i}}\right)$ in the unit square of densities (Fig. 7).

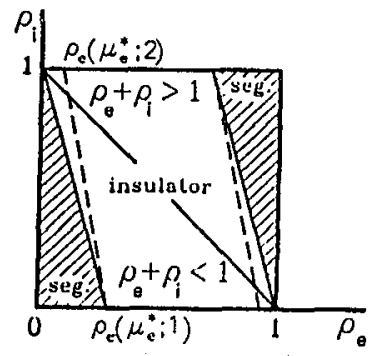

Fig. 7. The square of densities $\rho_{\mathrm{e}}$ and $\rho_{\mathrm{i}}$. In any state $\left(\rho_{\mathrm{e}}, \rho_{\mathrm{i}}\right)$ belonging to the hatched triangles the system is a conductor (no energy gap at the Fermi level) and the ground state configuration is the segregated one. For very small $U$ the hypotenuses of the hatched triangles may leave the square corners, so that the triangles transform into trapezoids whose boundaries are marked by the dashed lines. Between the triangles of the segregated configurations there extends the region where the ground-state configurations are (periodic and aperiodic) superstructures and the system is an insulator.

On the coexistence line of $c .1$ and $c .2$ neither the ion density nor the electron density are specified. While the ion density $\rho_{\mathrm{i}}$ can assume any value from the $[0,1]$ interval, the electron density $\rho_{\mathrm{e}}$ has to fulfill the inequality $0<\rho_{\mathrm{e}}<\overline{\rho_{\mathrm{e}}}\left(\rho_{\mathrm{i}}, U ; \mathcal{S}_{p}\right)$, where

$$
\overline{\rho_{\mathrm{e}^{\bullet}}}\left(\rho_{\mathrm{i}}, U ; \mathcal{S}_{p}\right)=\left(1-\rho_{\mathrm{i}}\right) \rho_{\mathrm{e}}\left(\mu_{\mathrm{e}}^{*}\left(\mathcal{S}_{p} ; U\right) ; 1\right)+\rho_{\mathrm{i}} \rho_{\mathrm{e}}\left(\mu_{\mathrm{e}}^{*}\left(\mathcal{S}_{p} ; U\right) ; 2\right),
$$

or the inequality

$$
\underline{\rho_{\mathrm{e}}^{* * *}}\left(\rho_{\mathrm{i}}, U ; \mathcal{S}_{p}\right)<\rho_{\mathrm{e}}<1,
$$

where

$$
\underline{\rho_{\mathrm{e}} \cdot}\left(\rho_{\mathrm{i}}, U ; \mathcal{S}_{p}\right)=1+\overline{\rho_{\mathrm{e}}^{*}}\left(\rho_{\mathrm{i}}, U ; \mathcal{S}_{p}\right)-\rho_{\mathrm{e}}\left(\mu_{\mathrm{e}}^{*}\left(\mathcal{S}_{p} ; U\right) ; 1\right)-\rho_{\mathrm{e}}\left(\mu_{\mathrm{e}}^{*}\left(\mathcal{S}_{p} ; U\right) ; 2\right) .
$$

The extremal electron densities $\overline{\rho_{\mathrm{e}^{*}}}$ and $\rho_{\mathrm{e}}^{* *}$ are, respectively, the upper and lower bounds for their $\mathcal{S}_{\infty}$ counterparts $\rho_{\mathrm{e}}^{*}$ and $\rho_{\mathrm{e}}^{* *}$. All the states $\left(\rho_{\mathrm{e}}, \rho_{\mathrm{i}}\right)$ satisfying the above conditions (see Fig. 7) are realized as the so-called segregated configurations, where all the ions clump together. The domains of the segregated configurations extend along the vertical sides of the square of densities, for all $0<\rho_{\mathrm{i}}<1$ (cf. Fig. 7). Therefore, for all $\rho_{\mathrm{i}}, 0<\rho_{\mathrm{i}}<1, U>0$, and for sufficiently small $\rho_{\mathrm{e}}$ $\left(\rho_{\mathrm{e}}<\overline{\rho_{\mathrm{e}}}\left(\rho_{\mathrm{i}}, U ; \mathcal{S}_{p}\right)\right)$ or sufficiently large $\rho_{\mathrm{e}}\left(\rho_{\mathrm{e}}>\underline{\rho_{\mathrm{e}}^{* * *}}\left(\rho_{\mathrm{i}}, U ; \mathcal{S}_{p}\right)\right)$ the g.s.c. is the segregated configuration. This is the content of the segregation principle which was formulated, for large $U$, as a conjecture for the $\mathcal{S}_{\infty}$-case and observed in canonical 
r.p.d. by Freericks and Falicov [1]. Alternatively, the segregation principle can be formulated as follows: for any $0<\rho_{\mathrm{i}}<1$ and $\rho_{\mathrm{e}}<1-\rho_{\mathrm{i}}\left(\rho_{\mathrm{e}}>1-\rho_{\mathrm{i}}\right)$ there is a $U_{\mathrm{cr}}$, depending only on the ratio $\rho_{\mathrm{e}} /\left(1-\rho_{\mathrm{i}}\right)\left(\rho_{\mathrm{i}} /\left(1-\rho_{\mathrm{e}}\right)\right)$, such that for $U>U_{\mathrm{cr}}$ the g.s.c. of the state $\left(\rho_{\mathrm{e}}, \rho_{\mathrm{i}}\right)$ is the segregated configuration.

In one dimension and for $U \gg U_{\text {sl }}$ the segregation principle has been proved: first by Gruber [2], when $\mathcal{S}$ consists of crenel configurations of arbitrary period and then by Lemberger [3] in the $\mathcal{S}_{\infty}$-case. The upper and lower bounds for $U_{\mathrm{cr}}$, when $U>U_{\mathrm{sl}}$, have been constructed by Brandt [12].

Proceeding with the translation from the grand canonical to the canonical ensemble, we see that the above mentioned properties of $\mu_{\mathrm{e}}^{*}\left(\mathcal{S}_{p} ; U\right)$ as a function

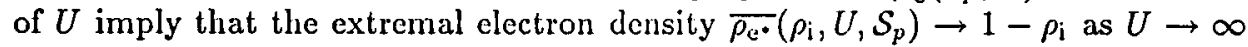
and $\overline{\rho_{\mathrm{e}^{\bullet}}}\left(\rho_{\mathrm{i}}, U, \mathcal{S}_{p}\right) \rightarrow 0$ as $U \rightarrow 0$. Moreover,

$$
U_{\text {cr }}\left(\frac{\rho_{\mathrm{e}}}{1-\rho_{\mathrm{i}}} ; \mathcal{S}_{p}\right) \rightarrow \infty \quad \text { as } \quad \frac{\rho_{\mathrm{e}}}{1-\rho_{\mathrm{i}}} \rightarrow 1
$$

and

$$
U_{\text {cr }}\left(\frac{\rho_{\mathrm{e}}}{1-\rho_{\mathrm{i}}} ; \mathcal{S}_{p}\right) \rightarrow 0 \text { as } \frac{\rho_{\mathrm{e}}}{1-\rho_{\mathrm{i}}} \rightarrow 0 .
$$

As long as $U$ is such that $\mu_{\mathrm{e}}^{*}(\mathcal{S} ; U)<U-2$ (i.e. large enough) the domains of the segregated configurations have the form of right-angled triangles whose bases are the vertical sides of the square of densities (Fig. 7). One of the triangles is located below the main diagonal $\rho_{\mathrm{e}}+\rho_{\mathrm{i}}=1$, the other one above it. For sufficiently small $U$ it may happen that $\mu_{\mathrm{e}}^{*}\left(\mathcal{S}_{p} ; U\right)>U-2$ (cf. Fig. 2). Then the domains of the segregated configuration assume the form of a right-angled trapezoid bounded by three sides of the square of densities. Thus for sufficiently small $U$ the domain of the segregated configurations may contain the small segments of the main diagonal that stick to the corners (cf. Fig. 7).

\section{The fine structure, $U$ large}

For the above description of the coarse structure of the phase diagram the qualitative knowledge of the band structures of the only two translationally invariant configurations (c.1 and c.2) was sufficient. On the contrary, for any attempt to understand the fine structure of the $\mathcal{S}_{p}$-r.p.d.'s the knowledge of the band structures of many translationally noninvariant configurations is indispensable. For this purpose the results obtained by Lyżwa [14] have been very useful. A part of the information collected, namely the positions of the gaps in the spectrum of the $\mathcal{S}_{6}$-r.g.s.c.'s and the electron densities in these gaps can be read in Figs. 2-6.

We start describing the fine structure of the $\mathcal{S}_{p}$-r.p.d. with the domains $d_{3}\left(\mathcal{S}_{p}\right)$ which are the upper bounds for $d_{3}\left(\mathcal{S}_{\infty}\right)$. These domains occupy the central part (around the origin $\mu_{\mathrm{e}}=\mu_{\mathrm{i}}=0$ ) of the domains $\mathcal{D}\left(\mathcal{S}_{p}\right)$ with $p \geq 2$ and are invariant with respect to the inversion about the origin. When $p$ increases, the domains $d_{3}\left(\mathcal{S}_{p}\right)$ decrease. IIowever, they shrink neither to the empty set nor to a point (the origin). It has been proved [13] that there is a polygon $\underline{d}_{3}$ around $\mu_{\mathrm{e}}=\mu_{\mathrm{i}}=0$ (shown as the cross-hatched region in the displayed diagrams), where $G$ consists of c.3, i.e. $\underline{d}_{3}$ is a lower bound for $d_{3}\left(\mathcal{S}_{p}\right)$. For a $U$ which is larger than $U_{\mathrm{sl}}$, at least 2,3 times, $d_{3}\left(\mathcal{S}_{p}\right)$ are cigar-like regions extending along $\gamma$. The 
projection $d_{3}^{\mathrm{e}}\left(\mathcal{S}_{p}\right)$ of $d_{3}\left(\mathcal{S}_{p}\right)$ onto the $\mu_{\mathrm{e}}$-axis is well contained in the only gap of c.3, i.e. in the interval $[-U, U]$ (cf. Fig. 6a). Thus in $d_{3}(\mathcal{S})$, where $\mathcal{S}=\mathcal{S}_{p}$ or $\mathcal{S}=\mathcal{S}_{\infty}$, the system is an insulator with the electron density $\rho_{\mathrm{e}}=\rho_{\mathrm{i}}=\frac{1}{2}$. The domain $d_{3}\left(\mathcal{S}_{p}\right)$ depends weakly on $p$ : by adding new configurations, with larger period, the border of $d_{3}\left(\mathcal{S}_{2}\right)$ becomes only somewhat thicker (therefore, $d_{3}\left(\mathcal{S}_{2}\right)$ might already be a good approximation to $\left.d_{3}\left(\mathcal{S}_{\infty}\right)\right)$. In a neighlborhood of the point $\left(\mu_{\mathrm{e}}^{*}, f\left(\mu_{\mathrm{e}}^{*}\right)\right)$ the structure of the border of $\mathcal{D}\left(\mathcal{S}_{6}\right)$ is shown in the dashed circle in Fig. $6 \mathrm{~b}$.

Starting inside $d_{3}\left(\mathcal{S}_{6}\right)$ and going up a vertical line with $-U+2<\mu_{\mathrm{e}}<U-2$, we observe the domains of the classes $3,10,5,8,14,22-$ the upper sequence. Going down such a line, the adjoint sequence of classes of configurations (the lower sequence), where any $S$ in a class $x$ is replaced by $-S$, is seen. This result is in perfect agreement with the predictions made on the basis of a perturbation expansion in $1 / U$ [15]. The ion densities in the upper (lower) sequence constitute an increasing (decreasing) finite sequence of rationals $r_{n}=q_{n} / p_{n}$, where $q_{n}$ and $p_{n}$ are relatively prime and $p_{n}$ is equal to the period of the configurations with the density $\rho_{\mathrm{i}}=r_{n}$. In the $\mathcal{S}_{2}$-r.p.d. these sequences are $\left\{\frac{1}{2}, \frac{1}{1}\right\},\left\{\frac{1}{2}, \frac{0}{1}\right\}$ and the sequences in the $\mathcal{S}_{p+1}$-r.p.d. are obtained from those in the $\mathcal{S}_{p}$-r.p.d. by inserting between the two consecutive rationals $q_{n} / p_{n}, q_{n+1} / p_{n+1}$ the new one: $\left(q_{n}+q_{n+1}\right) /\left(p_{n}+p_{n+1}\right)$ [16]. For instance, in the $\mathcal{S}_{6}$-r.p.d. the upper sequence of the ion densities is: $1 / 2,3 / 5,2 / 3,4 / 5,1$. Extrapolating to the $\mathcal{S}_{\infty}$-case, we are led to conjecture, as Barma and Subrahmanyam [15], that for each rational $q / p$, with $q$ and $p$ relatively prime, there is a domain of a class with $\rho_{\mathrm{i}}=q / p$ and period $p$. A similar conclusion has been drawn in the two-dimensional case [4].

Further properties of the fine structure can be deduced by examining the band structures of periodic configurations. For the range of $U$, just considered, it is readily seen that whatever periodic configuration we choose, a typical point from the interval $[-U-2, U+2]$ belongs to a gap. If $\rho_{\mathrm{i}}=q / p$, then the $(p-q)$-th gap, counting from the bottom of the spcctrum, is the largest one. The size of the largest gaps is comparable to $2 U$, which is the size of the gap of c.3, and they have large overlap with the latter gap. The electron density in these gaps is $1-q / p$ [14]. The projections onto the $\mu_{\mathrm{e}}$-axis of the domains of the upper and lower sequences are contained in the largest gaps of the corresponding configurations. Therefore, in the domains of the upper and lower sequences the system is an insulator and the neutrality condition $\rho_{\mathrm{e}}+\rho_{\mathrm{i}}=1$ is satisfied (when $U<0$, this condition is $\rho_{\mathrm{e}}=\rho_{\mathrm{i}}$, hence the name). The configurations which appear in the above sequences have been proved to be g.s.c.'s of a two-state lattice gas with purely repulsive, long-range and convex two-body interaction [17]. Thus, in the neutral case $\left(\rho_{\mathrm{e}}+\rho_{\mathrm{i}}=1\right)$ the effective interaction between the ions in the Falicov-Kimball model can be regarded as being of this kind*. The configurations belonging to the two above mentioned sequences have been named the most homogeneous configurations and an elegant method of constructing them has been proposed by Lemberger [3]. Moreover, Lemberger has proved that for sufficiently large $U$ it is the most homogeneous class that is the g.s.c. of the neutral $\left(\rho_{\mathrm{e}}+\rho_{\mathrm{i}}=1\right)$ state $\left(\rho_{\mathrm{e}}, \rho_{\mathrm{i}}\right)$.

\footnotetext{
*We thank Ch. Gruber for this remark.
} 
To a large extent the fine structure can be described in terms of the projections $d_{x}^{e}\left(\mathcal{S}_{6}\right)$, where $x$ is from the upper or lower sequence of classes. Not only the projections $d_{x}^{\mathrm{e}}\left(\mathcal{S}_{p}\right)$ of the classes with $\rho_{\mathrm{i}}=q / p$ are contained in the $(p-q)$-th gaps but their positions are correlated with the positions of the gaps. In particular, the overlap of two domains $d_{x}\left(\mathcal{S}_{6}\right), d_{x^{\prime}}\left(\mathcal{S}_{6}\right)$, measured in terms of the intersection $d_{x}^{\mathrm{e}}\left(\mathcal{S}_{6}\right) \cap d_{x^{\prime}}^{\mathrm{e}}\left(\mathcal{S}_{6}\right)$, is similar to the intersection of the corresponding gaps.

Moreover, we see in the dashed circle in Fig. 6b that the less dense the ions, the more is shifted to the right, the left end of a domain with $\rho_{\mathrm{i}}<1 / 2$ towards the upper edge of the band of c.1. In the configuration independent gap $[-U+2, U-2]$ the borders of domains are parallel, with the slope equal to 1 [4]. The vertical lines with $\mu_{\mathrm{e}} \in[-U+2, U-2]$ intersect all domains belonging to the upper and lower sequences.

We suggest that in the $\mathcal{S}_{\infty}$-case any vertical line from the stripe $-U+2<$ $\mu_{\mathrm{e}}<U-2$ intersects infinitely many domains of classes whose ion density $\rho_{\mathrm{i}}$ can be any arbitrary rational number. In those domains the electron density is well defined and the neutrality condition $\rho_{\mathrm{e}}+\rho_{\mathrm{i}}=1$ is satisfied. The neutral states with $\rho_{\mathrm{i}}$ irrational are represented by the borders between domains of the classes with rational $\rho_{\mathrm{i}}$. In all states satisfying the neutrality condition (the main diagonal in Fig. 7) the system is an insulator (see also a related remark by Freericks and Falicov [1] and the references they quote in connection with this remark).

For the range of $U$ considered, the non-neutral states, which are located between the main diagonal and the triangles of the segregated configurations (cf. Fig. 7 ), are not visible in the $\mathcal{S}_{6}$-r.p.d.'s. $A n$ explanation of this effect is provided in the next section.

\section{The fine structure, towards small $U$}

When $U$ decreases, the intervals which constitute the intersections of the gaps in which the configurations of the upper and lower sequences are g.s.c.'s decrease also. The larger is the difference of the ion densities between the two configurations, the larger is $U$ at which the intersections of these gaps become void. Consequently, the overlaps between the domains of configurations decrease. The transformation of the topology of a $\mathcal{S}_{p}$-r.p.d. can be described by means of a straight line that passes through the origin and intersects all the domains of the upper and lower sequence. When $U$ decreases, this line rotates counter-clockwise. Thus, well below $U_{\mathrm{s} l}$ the upper and lower sequences (the neutral sequences) of domains become, with respect to $d_{3}\left(\mathcal{S}_{p}\right)$, left and right sequences, respectively.

For $U<U_{\text {sl }}$ two new effects can immediately be noticed in the $\mathcal{S}_{6}$-r.p.d.'s. First, starting around $U=1$, new domains, not belonging to the two neutral sequences, appear. In the displayed lower halves of the r.p.d.'s these are, for instance, the domains of the classes $6,12,13,15,17$. Moreover, the domains of the configurations adjoint to the configurations of the upper sequence, that is, related to the latter by exchanging the holes with the ions, open (cf. Figs. 2 and 3). The new domains are located "south-west" with respect to the domains of the upper sequence. Second, for sufficiently small $U$ the intervals $d_{x}^{\mathrm{e}}\left(\mathcal{S}_{p}\right)$ which for larger $U$ have been contained in certain gaps start intersecting the neighboring bands. 
Thus, the electron density $\rho_{\mathrm{e}}$ is no longer a constant in the domains $d_{x}^{\mathrm{e}}\left(\mathcal{S}_{p}\right)$. This effect is most clearly seen in the case of $d_{3}^{e}\left(\mathcal{S}_{p}\right): d_{3}^{e}\left(\mathcal{S}_{6}\right) \cap[-U, U]$ becomes non-void slightly below $U=0.5$ (see Figs. 2 and 3). The same effect takes place in the canonical restricted phase diagrams [1].

In the sequel we shall argue that the two effects mentioned above are caused by the finiteness of the set $\mathcal{S}_{p}$ of admissible configurations. If $U$ is not too small, the projections onto the $\mu_{\mathrm{e}}$-axis of the new domains, that do not belong to the neutral sequences, are also contained in gaps of their configurations but $\rho_{\mathrm{e}}+\rho_{\mathrm{i}}<1$. For instance, in $d_{6}\left(\mathcal{S}_{6}\right) \rho_{\mathrm{e}}=\frac{1}{4}$, in $d_{15}\left(\mathcal{S}_{6}\right) \rho_{\mathrm{e}}=\frac{1}{6}$, in $d_{17}\left(\mathcal{S}_{6}\right) \rho_{\mathrm{e}}=\frac{1}{6}$, in $d_{9}\left(\mathcal{S}_{6}\right)$ $\rho_{\mathrm{e}}=\frac{2}{5}$ (cf. Fig. 3). This observation is consistent with the canonical restricted phase diagrams [1]: for $\rho_{\mathrm{i}}=\frac{1}{2}$ and $U$ not too close to zero the classes 6 and 15 are g.s.c.'s with the same electron densities as in our diagrams. Moreover, it explains why the domains of the non-neutral states (i.e. $\rho_{\mathrm{e}}+\rho_{\mathrm{i}} \neq 1$ ) are missing in the $\mathcal{S}_{p}$-r.p.d. for large $U$. Namely, all the states $\left(\rho_{\mathrm{e}}\left(S^{\prime}\right), \rho_{\mathrm{i}}(S)\right)$ which correspond to configurations $S \in \mathcal{S}_{p}$, where $\rho_{e}(S)$ stands for the electron density in such a gap of $S$ that $\rho_{\mathrm{e}}(S)+\rho_{\mathrm{i}}(S) \neq 1$, are contained in the triangular regions where the segregated configurations are the g.s.c.'s (Fig. 7). The larger is $\mathcal{S}_{p}$, the larger is $U$ below which domains with $\rho_{\mathrm{e}}+\rho_{\mathrm{i}} \neq 1$ can appear in the $\mathcal{S}_{p}$-r.p.d.

Contrary to the first effect, the second one seems to be solely a side effect of the $\mathcal{S}_{p}$-r.p.d., i.e. it is expected to disappear in the $\mathcal{S}_{\infty}$-case. When $p$ grows, all the domains and the intersections of the intervals $d_{x}^{e}\left(\mathcal{S}_{p}\right)$ with the bands decrease rapidly.

In the case of c.3 we can estimate the value of $U$ above which $\rho_{\mathrm{e}}=\frac{1}{2}$ in the whole domain $d_{3}\left(\mathcal{S}_{\infty}\right)$. As has been shown by Gruber et al. [4], for any configuration $S$ there is a point $\left(\mu_{\mathrm{e}}(S), \mu_{\mathrm{i}}(S)\right)$ on $\gamma_{1,2}$ where the energy $e\left(\mu_{\mathrm{e}}(S), \mu_{\mathrm{i}}(S) ; S\right)$ coincides with the energies of $c .1$ and c.2. The domain of the configuration $S$ has to be inside the rectangle $\mathcal{R}(S)$ built on the points $\left(\mu_{\mathrm{e}}(S), \mu_{\mathrm{i}}(S)\right)$ and $\left(-\mu_{\mathrm{e}}(-S),-\mu_{\mathrm{i}}(-S)\right)$ (this rectangle is contained in $\mathcal{R}$ ). In the one-dimensional case, using the exact densities of states of c.1, c.2, and c.3 [14], we found that the equations $e\left(\mu_{\mathrm{e}}, \mu_{\mathrm{i}} ; 1\right)=$ $e\left(\mu_{\mathrm{e}}, \mu_{\mathrm{i}} ; 2\right)=e\left(\mu_{\mathrm{e}}, \mu_{\mathrm{i}} ; 3\right)$ and $\mu_{\mathrm{e}}=U$ are equivalent to the following equation with respect to $U$ :

$$
\sqrt{U^{2}+4} \mathcal{E}\left(2 / \sqrt{U^{2}+4}\right)-\sqrt{1-U^{2}}-U \arcsin U=1
$$

where $\mathcal{E}(\cdot)$ stands for the complete elliptic integral of the second kind. Equation (7) can be solved numerically with arbitrary accuracy: $U=0.36 U_{\mathrm{sl}}$ to two significant figures. If $U>0.36 U_{\mathrm{sl}}$, the rectangle $\mathcal{R}(S)$ is contained in the stripe $-U<\mu_{\mathrm{e}}<U$, hence $d_{3}^{\mathrm{e}}\left(\mathcal{S}_{\infty}\right) \subset[-U, U]$, i.e. thie clectron density is constant in $d_{3}\left(\mathcal{S}_{\infty}\right)$. On the basis of the above remarks we think that the electron density in any domain $d_{x}\left(\mathcal{S}_{\infty}\right) \subset \mathcal{D}\left(\mathcal{S}_{\infty}\right)$ is constant for all $U$.

Therefore, we conjecture that in all the domains contained in $\mathcal{D}\left(\mathcal{S}_{\infty}\right)$ the system is an insulator. The system is of course a conductor in these parts of the c.1 and c.2 domains where the band is partially filled. Aside of these regions, the system is a conductor only on the coexistence line $\gamma_{1,2}$, or equivalently, in the states belonging to the domains of the segregated phases (Fig. 7). 


\section{Towards the complete phase diagram}

Of course, the data available in the $\mathcal{S}_{p}$-r.p.d. with $p \leq 6$ are not sufficient to reveal many of the propertics of the fine structure. Nevertheless, we tried to conceive the general structure of the $\mathcal{S}_{\infty}$-phase diagram. Namcly, we made an attempt to map the diagram in Fig. 7 onto the $\left(\mu_{\mathrm{e}}, \mu_{\mathrm{i}}\right)$-plane by displaying the regions of constant ion density. The results for a large $U$ (above $U_{\mathrm{sl}}$ ) and for a small $U$ (well below $U_{\mathrm{sl}}$ ) are shown in Figs. 8 and 9, respectively. To each rational

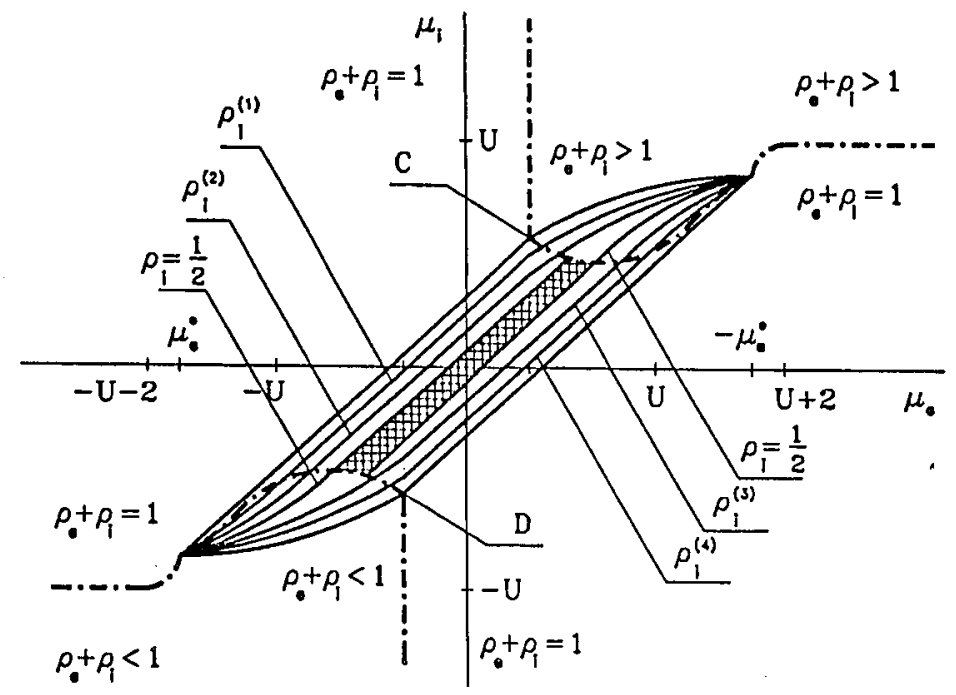

Fig. 8. The conjectured general structure of the pliase diagram in the $\mathcal{S}_{\infty}$-case and for $U>U_{\text {sl }}$. The continuous lines mark the borders of $\mathcal{D}\left(\mathcal{S}_{\infty}\right)$ and five lines of constant ion density corresponding to $\rho_{\mathrm{i}}^{(1)}>\rho_{\mathrm{i}}^{(2)}>1 / 2>\rho_{\mathrm{i}}^{(3)}>\rho_{\mathrm{i}}^{(1)}$. The dash-dotted lines divide the $\left(\mu_{\mathrm{e}}, \mu_{\mathrm{i}}\right)$-plane into three regions, where $\rho_{\mathrm{e}}+\rho_{\mathrm{i}}$ is smaller than 1 , equal to 1 , and greater than 1. The neutral region, where $\rho_{\mathrm{e}}+\rho_{\mathrm{i}}=1$, extends between the dash-dotted lines. Correspondingly, every constant density line is divided into three segments being the intersections of this line with the regions mentioned. The internal segment of the $\rho_{\mathrm{i}}=1 / 2$ line (contained in the neutral region) is the c.3-domain (the hatched region). The points $C$ and $D$ are the intersections of the boundary of $\mathcal{D}\left(\mathcal{S}_{\infty}\right)$ with the lines $\mu_{\mathrm{e}}=U-2$ and $\mu_{\mathrm{e}}=-U+2$, respectively. The whole structure of the pliase diagram follows the counter-clockwise rotation of the line connecting $C$ with $D$ when $U$ decreases.

number $0<\rho_{\mathrm{i}}<1$ there corresponds one stripe which represents a sequence of domains, as $\mu_{\mathrm{e}}$ varies, of different periodic configurations with the same ion density. Because of the scale adopted, these stripes are shown as lines, except the case $\rho_{\mathrm{i}}=\frac{1}{2}$, since this particular stripe is much wider than the others.

A line of constant density starts at $\left(\mu_{\mathrm{e}}^{*}, f\left(\mu_{\mathrm{e}}^{*}\right)\right)$ and ends at $\left(-\mu_{\mathrm{e}}^{*}, f\left(-\mu_{\mathrm{e}}^{*}\right)\right)$. It consists of three segments: two external oncs, being the intersections of the line of constant density with the non-neutral regions, and the internal one, being the intersection of this line with the neutral region. The internal segment of any con- 


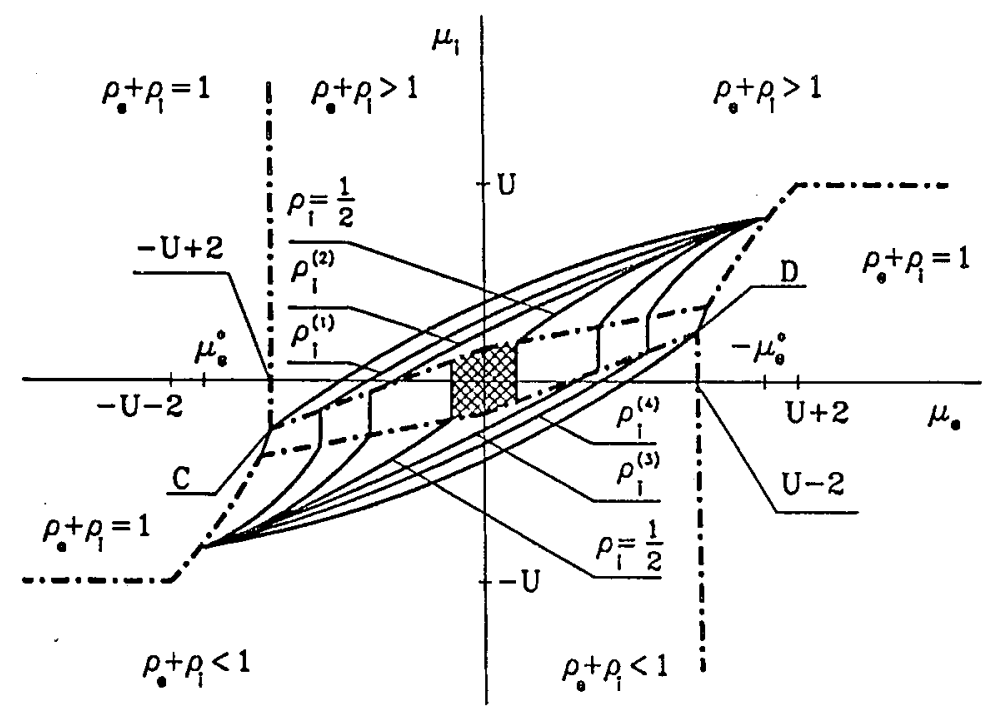

Fig. 9. The conjectured general structure of the pliase diagram in the $S_{\infty}$-case and for $U$ small (well below $U_{\mathrm{sl}}$ ). See the caption of Fig. 8 for the details.

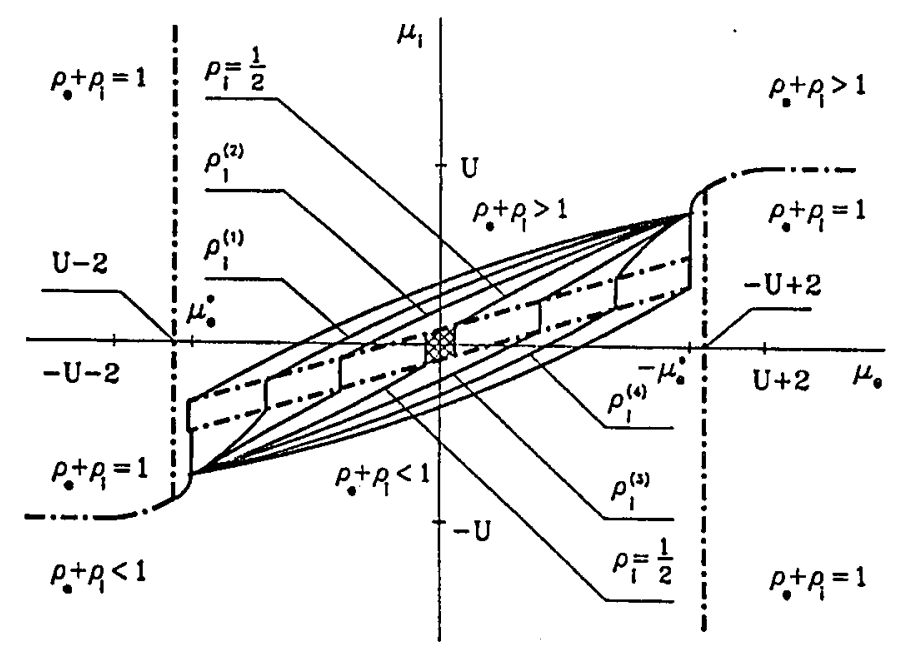

Fig. 10. The hypothetical structure of the pliase diagram for sufficiently small $U$. For the legend see the caption of Fig. 8. Contrary to the diagrams displayed in Figs. 8 and 9, here the neutral region is not connected.

stant density line is the domain of a one most homogeneous class (i.e. belonging to one of the neutral sequences). The two external segments represent two countably infinite sequences of domains of periodic non-neutral classes. The qualitative loca- 
tion of the borders between the neutral states and the non-neutral ones is sketched in Figs. 8 and 9 by means of the dash-dotted line. The restricted phase diagrams constructed by Freericks and Falicov [1] give us an idea of the types of domains which appear along the lines corresponding to $\rho_{\mathrm{i}}=\frac{1}{2}, \rho_{\mathrm{i}}=\frac{1}{3}$, and $\rho_{\mathrm{i}}=\frac{2}{3}$.

In view of the hypotheses put forward above, when $U$ decreases, a system initially in any non-neutral state $\left(\rho_{\mathrm{e}}, \rho_{\mathrm{i}}\right)$, undergoes a conductor-insulator transition at $U_{\mathrm{cr}}\left(\rho_{\mathrm{e}} /\left(1-\rho_{\mathrm{i}}\right)\right)$. If $U>U_{\mathrm{cr}}$, the system is a conductor and the ions' configuration is the segregated one. If $U<U_{\mathrm{cr}}$, the system is an insulator and the ions form a periodic (if $\rho_{\mathrm{i}}$ is rational) or an aperiodic (if $\rho_{\mathrm{i}}$ is irrational) configuration, where not all the distances between the nearest-neighbor ions are minimal (a superstructure). The value of $U_{\mathrm{cr}}$ is determined by the condition that one of the hypotenuses of the triangles of the segregated configurations (Fig. 7) passes through the point $\left(\rho_{\mathrm{e}}, \rho_{\mathrm{i}}\right)$.

When $U$ is much larger than $U_{\text {sl }}$, a typical state $\left(\rho_{e}, \rho_{\mathrm{i}}\right)$ is conducting while the insulating states are located in a narrow parallelogram whose diagonal coincides with the main diagonal of the square of densities. If $U$ is smaller than $U_{\text {sl }}$, a typical state $\left(\rho_{\mathrm{e}}, \rho_{\mathrm{i}}\right)$ is insulating while the conducting states occupy small triangular regions extending along the two vertical sides of the square of densities.

If, as has been noted in Sec. 3, for sufficiently small $U$ the triangles of the segregated configurations transform into trapczoids (Fig. 7), then the opposite transitions, i.e. insulator-conductor, occur when $U$ decreases. In particular, some neutral states which are located in a neighborhood of the corners $(0,1)$ and $(1,0)$ of the square of densities (Fig. 7) become conducting. The possibility of loosing stability by some most homogeneous configurations for sufficiently small $U$ has been suggested by Gruber [18] on the basis of a quantum-mechanical argument. Consequently, in the $\left(\mu_{\mathrm{e}}, \mu_{\mathrm{i}}\right)$-phase diagrams the ends of the neutral sequences, i.e. the domains of the most homogencous configurations with $\rho_{\mathrm{i}}$ close to 1 or to 0 disappear. This situation is represented in the qualitative phase diagram (Fig. 10) similar to those in Figs. 8 and 9.

Further properties of the phase diagram of the Falicov-Kimball model are under investigation. Also the implications of the diagrams proposed here for the theory of mixed-valence phenomena are being examined. The results will be reported elsewhere.

\section{Acknowledgments}

One of the authors (J.J.) has benefited from discussions with Ch. Gruber and P. Lemberger during his visit at Institut de Physique Théorique of the Ecole Polytechnique Fédérale de Lausanne. It is a pleasure to thank them for interesting discussions and warm hospitality. We are also gratcful to our colleagues M.R. Dudek for his kindly helping us in the printing out the last three figures and to $\mathrm{K}$. Rapcewicz for reading the manuscript. 


\section{References}

[1] J.K. Freericks, L.M. Falicov, Phys. Rev. B 41, 2163 (1990).

[2] Ch. Gruber, Helv. Phys. Acla 64, 668 (1991).

[3] P. Lemberger, J. Phys. A 25, 715 (1992).

[4] Ch. Gruber, J. Jędrzejewski, P. Lemberger, J. Stat. Phys. 66, 913 (1992).

[5] J. Jędrzejewski, J. Lach, R. Eyżwa, Phys. Lell. A 134, 319 (1989).

[6] J. Jędrzejewski, J. Lach, R. Lyżwa, Physica A 154, 529 (1989).

[7] L.M. Falicov, J.C. Kimball, Phys. Rev. Lett. 22, 997 (1969).

[8] D.I. Khomskii, in: Quantum Theory of Solids, Ed. I.M. Lifshits, Mir, Moskva 1982, p. 70 .

[9] G. Czycholl, Phys. Rep. 143, 277 (1986).

[10] T. Kennedy, E.H. Lieb, Physica A 138, 320 (1986).

[11] U. Brandt, R. Schmidt, Z. Phys. B 63, 45 (1986).

[12] U. Brandt, J. Low Temp. Phys. 84, 177 (1991).

[13] Ch. Gruber, J. Iwański, J. Jędrzejewski, P. Lemberger, Phys. Rev. B 41, 2198 (1990).

[14] R. Łyżwa, Phys. Lett. A 164, 323 (1992); Physica A 102, 231 (1993).

[15] M. Barma, V. Subrahmanyam, Phase Transit. B 16, 303 (1989).

[16] S. Aubry, J. Phys. Lell. (France) 44, L-247 (1983).

[17] J. Ilubbard, Phys. Rev. B 17, 494 (1978).

[18] Ch. Gruber, private communication.

[19] J.K. Freericks, Phys. Rev. B 47, 9263 (1993).

[20] J. Lach, R. Eyżwa, J. Jędrzejewski, preprint ITP UWr 804/92, Institute of Theoretical Physics, University of Wroclaw. 